\title{
Enhanced Adsorption of Orange II Using Cationic Surfactant Modified Biochar Pyrolyzed from Cornstalk
}

\author{
Xiao Mi, Guoting Li, Weiyong Zhu, and Lili Liu \\ Department of Environmental and Municipal Engineering, North China University of Water Resources and Electric Power, \\ Zhengzhou 450011, China
}

Correspondence should be addressed to Guoting Li; lipsonny@163.com

Received 31 December 2015; Accepted 18 April 2016

Academic Editor: Marisol Belmonte

Copyright (C) 2016 Xiao Mi et al. This is an open access article distributed under the Creative Commons Attribution License, which permits unrestricted use, distribution, and reproduction in any medium, provided the original work is properly cited.

\begin{abstract}
As dissolution of raw biomass is serious when used as an adsorbent, the cheap biochar pyrolyzed from biomass might be a good matrix. Raw cornstalk biochar was intentionally modified by cetyltrimethylammonium bromide (CTAB) to prepare the composite adsorbent designed for the removal of negatively charged pollutants. After modification, the removal efficiency for anionic dye Orange II (ORII) increased from $46.9 \%$ of the virgin cornstalk biochar to $99.7 \%$ of the CTAB-modified cornstalk biochar. The uptake of ORII proved to be favorable under acidic conditions but unfavorable under alkaline conditions. By nonlinear simulation, the Elovich model was the best to describe the adsorption kinetics. For linear simulation of the kinetic data, the pseudo-secondorder kinetic model fitted the experimental points better than the pseudo-first-order model. Kinetic analysis indicated that the ORII adsorption process on the CTAB-modified cornstalk biochar might be chemical adsorption accompanied by ion exchange. At $298 \mathrm{~K}$, the maximal adsorption capacity of the modified biochar is $26.9 \mathrm{mg} / \mathrm{g}$ by the Langmuir model. The adsorption of ORII increased with a rise in the reaction temperature. The enthalpy and entropy of the adsorption process are calculated to be $38.45 \mathrm{KJ} \mathrm{mol}^{-1}$ and $185.0 \mathrm{~J} \mathrm{~mol}^{-1} \mathrm{~K}^{-1}$, respectively. The negative values of $\Delta G^{0}$ at 288,298 , and $308 \mathrm{~K}$ were $-14.92,-16.50$, and $-18.62 \mathrm{KJ} \mathrm{mol}^{-1}$, respectively. The above thermodynamic analysis demonstrates that the adsorption process was endothermic and spontaneous.
\end{abstract}

\section{Introduction}

An increasing number of organic pollutants, such as dyes, endocrine-disruptors, and pharmaceutical and personal care products, have been detected in the natural environment and wastewaters [1,2]. Accordingly, a number of techniques have been applied to remove these organic pollutants efficiently. Among these techniques, adsorption is currently considered as an effective, efficient, and economic method for water purification [3]. In recent years, some novel composite adsorbents have attracted great attention as they are particularly effective and efficient for the removal of particular pollutants $[4,5]$. As these composite materials integrate the properties and advantages of each of their components, they are intentionally designed to remove the target pollutants efficiently and cost-effectively. As a result, the exact design and application of the pollutants-oriented adsorbent seem to be a promising and practical approach for adsorbent development.
As we know, activated carbon is well accepted as the most widely used adsorbent for water purification around the world due to its high surface area, porous structure, and special surface reactivity [6]. However, considering the high expenditure for the application of activated carbon, low-cost adsorbents, such as agricultural by-product and industrial waste, have become the applicable matrix or constituent to prepare suitable adsorbents. However, most of the biomass is not used properly. Hence, utilization of these carbonaceous materials for the composite adsorbent preparation will be not only useful but also cost-effective.

As a kind of low-cost adsorbents, these biomass related adsorbents have been widely explored for the removal of a number of pollutants including dyes and heavy metals [7-9]. However, one significant disadvantage of the raw biomassbased sorbent is the dissolution of the raw biomass, which might lead to organic leaching and secondary pollution. Low temperature pyrolysis of biomass with carbon sequestration and gas capture is expected to be a carbon-neutral energy 
source [10]. The surface area of the resulting biochar is usually higher than the raw biomass. Biochar has potential applications in environmental management such as soil improvement, waste management, climate change mitigation, and energy production [11]. Consequently, there are a great need and possibility of combining biochar with other constituents to design composite adsorbents.

Regarding the adsorptive removal of water soluble dyes, Zhang and coworkers treated cornstalk with cetyltrimethylammonium bromide (CTAB), and the prepared sorbent was used for effectively removing an anionic dye Congo red [12]. As expected, preliminary study indicated that the raw cornstalk could definitely produce brown organic matters dissolved in aqueous media and cause secondary pollution. In this research, a stable cornstalk biochar pyrolyzed at low temperature $\left(600^{\circ} \mathrm{C}\right)$ was intentionally modified by CTAB to enhance its positive charge property. An anionic dye, Orange II, was selected as a target organic pollutant for the adsorption process.

\section{Materials and Methods}

2.1. Materials and Apparatus. Orange II (ORII, mass fraction $>95 \%)$, methylene blue (MB, mass fraction > 98.5\%, chemically pure), and cetyltrimethylammonium bromide (CTAB) were purchased from Beijing Chemical Reagents Company and used without further purification. Other chemicals used were of analytical grade. Deionized (DI) water was used throughout the study.

2.2. Preparation of CTAB-Modified Cornstalk Biochar. Cornstalk was collected from a farmland in Zhengzhou of Henan Province. The collected biomass was washed, dried, crushed, and sieved using a 40 mesh sieve. Then the cornstalk was pyrolyzed at $600^{\circ} \mathrm{C}$ for $3 \mathrm{~h}$ in a furnace under an oxygenlimited condition. The resultant biochar was demineralized in a $4 \mathrm{~mol} / \mathrm{L} \mathrm{HCl}$ solution for $12 \mathrm{~h}$ and separated by filtration. Then the residues were rinsed with deionized (DI) water to neutral solution $\mathrm{pH}$ and dried in an oven at $80^{\circ} \mathrm{C}$ overnight. One gram of the demineralized cornstalk biochar was added into $100 \mathrm{~mL}$ of CTAB solution (1.0\%). The mixture was shaken by an orbital shaker at $120 \mathrm{rpm}$ for $24 \mathrm{~h}$. Then the modified biochar was separated by filtration and dried at $60^{\circ} \mathrm{C}$ for $4 \mathrm{~h}$. Finally, the prepared CTAB-modified cornstalk biochar was stored in a desiccator for further use.

2.3. Characterization. The morphologies of raw cornstalk and cornstalk biochar were recorded on a Philips Quanta2000 scanning microscope coupled with an energy dispersive X-ray (EDX) spectrometer. FTIR spectra (KBr pellets) were recorded on a Nicolet NEXUS 470 FTIR spectrophotometer from 400 to $4000 \mathrm{~cm}^{-1}$.

2.4. Adsorption of Dye on the CTAB-Modified Cornstalk Biochar. The adsorption of ORII and MB on the CTAB-modified cornstalk biochar was conducted in a series of $100 \mathrm{~mL}$ conical flasks. For the tests of adsorption isotherm and $\mathrm{pH}$ effect, $20 \mathrm{mg}$ of CTAB-modified cornstalk biochar was added into
$50 \mathrm{~mL}$ of ORII solution with an initial concentration of $10 \mathrm{mg} / \mathrm{L}$. These flasks were shaken on a horizontal shaker for $24 \mathrm{~h}$ at a speed of $140 \mathrm{rpm}$. For the kinetics study, $400 \mathrm{mg}$ of CTAB-modified cornstalk biochar was added into $1000 \mathrm{~mL}$ of ORII solution with an initial concentration of $10 \mathrm{mg} / \mathrm{L}$. Constant stirring was maintained by a magnetic stirrer. Samples were collected at a desired time interval.

The temperature was controlled at a constant value of $298 \mathrm{~K}$ except for the study on the adsorption isotherm at different temperatures. All of the solution $\mathrm{pH}$ was maintained at neutral $\mathrm{pH}$ except for the $\mathrm{pH}$ effect study. The solution $\mathrm{pH}$ adjustment was conducted by adding diluted $\mathrm{HNO}_{3}$ or $\mathrm{NaOH}$ ( $2 \mathrm{~mol} / \mathrm{L})$ solution.

2.5. Analyses. Samples were collected and filtered through a $0.45 \mu \mathrm{m}$ membrane before being analyzed. The concentrations of ORII and MB were determined by measuring the maximum absorbance at a fixed wavelength of 484 and $664 \mathrm{~nm}$, respectively, using UVmini-1240 spectrophotometer (Shimadzu, Japan).

The removal efficiency of ORII was calculated as

$$
\text { Removal efficiency }=\left(1-\frac{C_{t}}{C_{0}}\right) \times 100 \%,
$$

where $C_{t}$ is the dye concentration at time $t$ and $C_{0}$ is the initial dye concentration.

The quantity of ORII adsorbed on the CTAB-modified cornstalk biochar was calculated by the following equation:

$$
\begin{aligned}
& q_{e}=\left(C_{0}-C_{e}\right) \frac{V}{W}, \\
& q_{t}=\left(C_{0}-C_{t}\right) \frac{V}{W},
\end{aligned}
$$

where $q_{e}$ and $q_{t}(\mathrm{mg} / \mathrm{g})$ are the adsorption capacity at equilibrium and $t$ min; $C_{0}$ is the initial concentration of ORII in solution, while $C_{e}$ and $C_{t}(\mathrm{mg} / \mathrm{L})$ are the concentrations of ORII at equilibrium and $t$ min, respectively; $V(\mathrm{~L})$ is the volume of solution and $W(\mathrm{~g})$ is the mass of adsorbent used.

\section{Results and Discussion}

3.1. Characterization of Cornstalk Biochar. As illustrated in Figure 1, both the raw cornstalk and the cornstalk biochar pyrolyzed at $600^{\circ} \mathrm{C}$ consist of big flakes within the size of $50 \mu \mathrm{m}$. The treatment by pyrolysis did not obviously alter the morphologies of the raw cornstalk except that the flakes became a little thinner. However, by the concurrent EDX analysis, the contents of $\mathrm{C}$ and $\mathrm{N}$ element increased from $60.5 \%$ and $3.1 \%$, respectively, of the raw cornstalk to $75.5 \%$ and $6.0 \%$, respectively, of the cornstalk biochar pyrolyzed at $600^{\circ} \mathrm{C}$. Besides, the content of $\mathrm{O}$ element decreased from $17.3 \%$ of the raw cornstalk to $6.9 \%$ of the cornstalk biochar. The above indicates that the functional groups and the polarity of the raw cornstalk were significantly changed.

The FTIR spectra of the raw biochar, the raw CTABmodified biochar, and the exhausted CTAB-modified biochar are recorded in Figure 2. Compared to the raw biochar, 


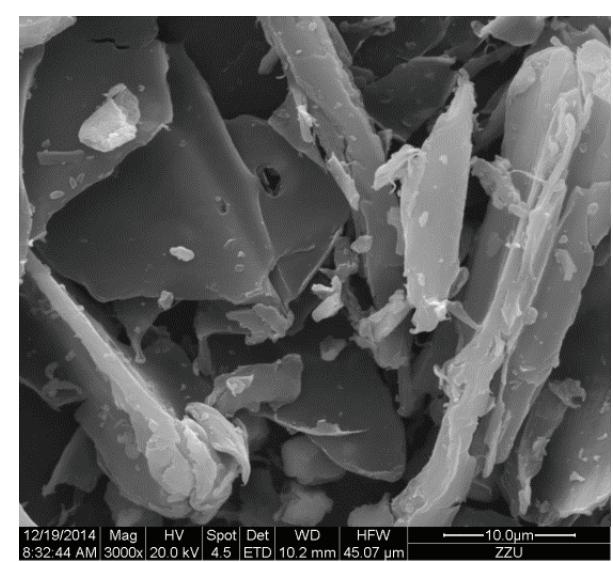

(a)

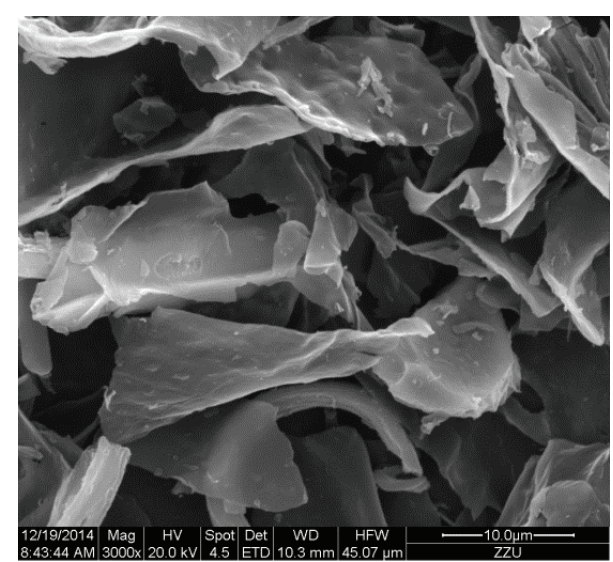

(b)

FIGURE 1: SEM image of raw cornstalk (a) and cornstalk biochar pyrolyzed at $600^{\circ} \mathrm{C}$ (b).

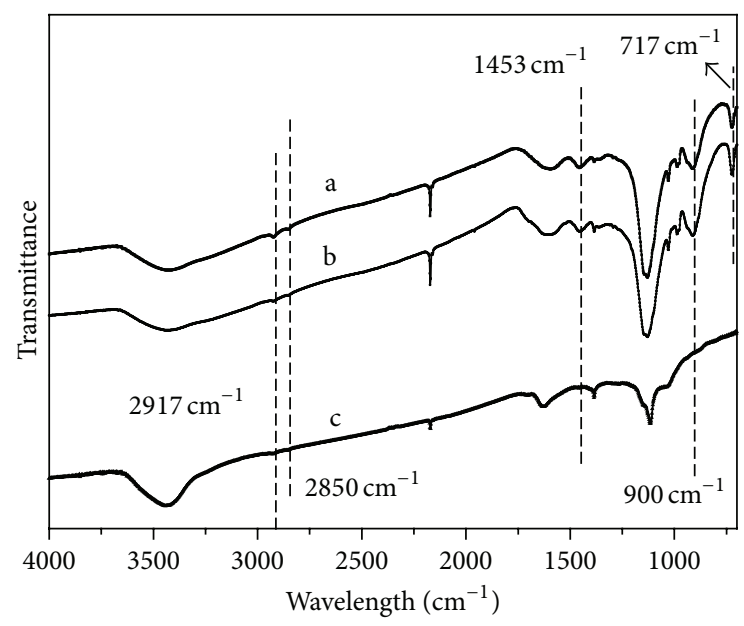

a: CTAB-biochar

b: exhausted CTAB-biochar

c: raw biochar

FIGURE 2: FTIR spectra of raw biochar, CTAB-modified biochar, and exhausted CTAB-modified biochar.

several new absorbance bands at 2917, 2850, 1453, 900, and $717 \mathrm{~cm}^{-1}$ appeared on the FTIR spectra of the raw CTABmodified biochar. The bands at 2917 and $2850 \mathrm{~cm}^{-1}$ are attributed to the symmetric and asymmetric stretching vibrations of aliphatic $-\mathrm{CH}_{2}$ in CTAB while those at $1453 \mathrm{~cm}^{-1}$ are attributed to the symmetric and asymmetric $\mathrm{C}-\mathrm{H}$ scissoring vibrations of a $\mathrm{CH}_{3}-\mathrm{N}^{+}$moiety. Also, the change in the $\mathrm{CH}_{2}$ rocking mode from a doublet at 730 and $719 \mathrm{~cm}^{-1}$ for pure CTAB to a singlet at $717 \mathrm{~cm}^{-1}$ for the CTABmodified biochar demonstrates the interaction between the $\mathrm{N}$-containing group and biochar [13]. Additionally, the bands of CTAB on the exhausted CTAB-modified biochar are as significant as those on the virgin CTAB-modified biochar, which also indicates the solid combination of CTAB with the biochar substrate. The above results indicate the good

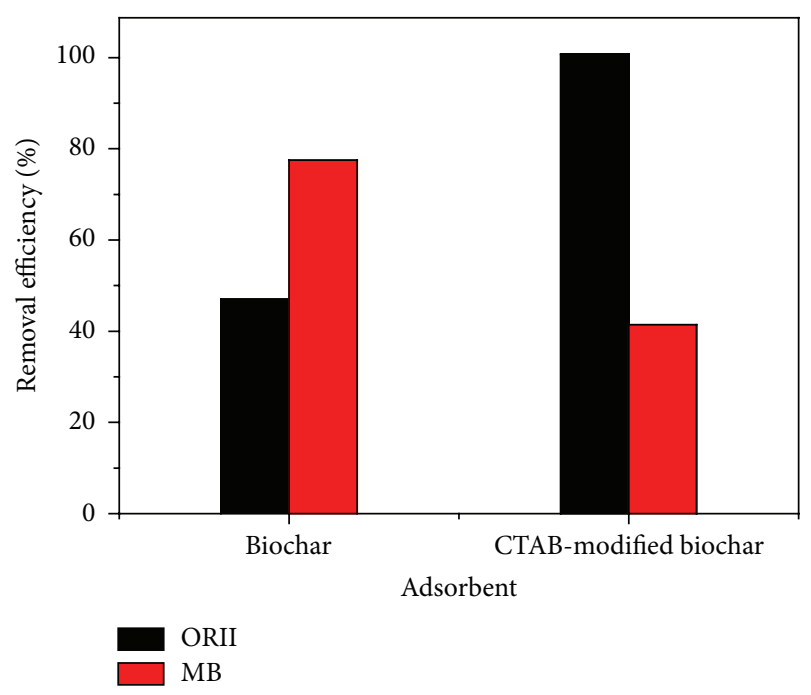

FIGURE 3: Effect of charge property of dye on the adsorption by CTAB-modified biochar. The concentration for both ORII and MB was $8 \mathrm{mg} / \mathrm{L}$, sorbent dosage $20 \mathrm{mg}$.

immobilization and interaction of CTAB onto the cornstalk biochar.

3.2. Effect of Charge Property of Dye on Adsorption. The immobilization of cationic surfactant CTAB onto cornstalk biochar is intended to modify the surface charge properties of the raw biochar, which is expected to facilitate the adsorptive removal of anionic pollutants. As a comparison, the adsorption of anionic dye ORII and cationic dye MB was investigated, as illustrated in Figure 3. The concentration for both dyes was $8 \mathrm{mg} / \mathrm{L}$ and the sorbent dosage was fixed at $20 \mathrm{mg}$. The removal efficiencies for ORII and MB on the raw cornstalk biochar were $46.9 \%$ and $77.6 \%$, respectively, while they achieved $99.7 \%$ and $41.5 \%$, respectively, on the CTAB-modified biochar. As the raw biochar surfaces are 
normally negatively charged [14], the positively charged $M B$ molecules are easy to adsorb onto the raw biochar while the uptake of the negatively charged ORII molecules is expected to be difficult due to electrostatic interaction. In contrast, the CTAB-modified cornstalk biochar is typically positively charged as a consequence of the immobilization of CTAB onto the biochar, which especially leads to the adsorption of ORII molecules while inhibiting significantly the uptake of MB molecules. As presented in Figure 3, almost all the ORII molecules were removed by the CTAB-modified cornstalk biochar, indicating the biochar surface is overwhelmingly positively charged. Apparently, the effect of electrostatic interaction is dominant during the adsorption process.

3.3. Effect of Adsorbent Dosage. As the CTAB-modified cornstalk biochar proved to be especially powerful for the adsorptive removal of ORII, the effect of the sorbent dosage was explored with an initial ORII concentration at 10 and $15 \mathrm{mg} / \mathrm{L}$, respectively. As presented in Figure 4, at the ORII concentration of $10 \mathrm{mg} / \mathrm{L}$, the removal efficiency increased from $18.7 \%$ at $5 \mathrm{mg}$ dosage to $98.3 \%$ at $40 \mathrm{mg}$ dosage. Even at the ORII concentration of $15 \mathrm{mg} / \mathrm{L}$ the removal efficiency increased from $8.1 \%$ at $5 \mathrm{mg}$ dosage to $97.2 \%$ at $40 \mathrm{mg}$ dosage. The above indicates that the CTAB-modified cornstalk biochar is capable of removing ORII efficiently. The dosage of the sorbent was fixed at $20 \mathrm{mg}$ in $50 \mathrm{~mL}$ solution while the ORII concentration was at $10 \mathrm{mg} / \mathrm{L}$ in the following experiments.

\subsection{Adsorption Kinetics under Different Solution pH Condi-} tions. Adsorption kinetics for ORII uptake on the CTABmodified cornstalk biochar was investigated at $\mathrm{pH}$ 3.0, 5.0, 7.0, 9.0, and 11.0, respectively. Three kinetic models including pseudo-first-order, pseudo-second-order, and Elovich models were used to fit the experimental data.

The mathematical representations of the linear and nonlinear models of pseudo-first-order and pseudo-secondorder kinetics are given in $[15,16]$

$$
\begin{aligned}
q_{t} & =q_{e}\left(1-e^{-k_{1} t}\right), \\
\ln \left(q_{e}-q_{t}\right) & =\ln q_{e}-k_{1} t, \\
q_{t} & =\frac{k_{2} q_{e}^{2} t}{\left(1+k_{2} q_{e} t\right)}, \\
\frac{t}{q_{t}} & =\frac{1}{k_{2} q_{e}{ }^{2}}+\frac{t}{q_{e}},
\end{aligned}
$$

where $q_{e}$ and $q_{t}$ are the adsorption capacities $(\mathrm{mg} / \mathrm{g})$ at equilibrium and at time $t(\mathrm{~min})$, respectively, and $k_{1}\left(\mathrm{~min}^{-1}\right)$ and $k_{2}$ (g/mg.min) are the related adsorption rate constants for the pseudo-first-order and the pseudo-second-order model, respectively.

Concurrently, the Elovich model was also used for the nonlinear simulation. The Elovich model can be written as $[17,18]$

$$
q_{t}=a \ln (t)+b
$$

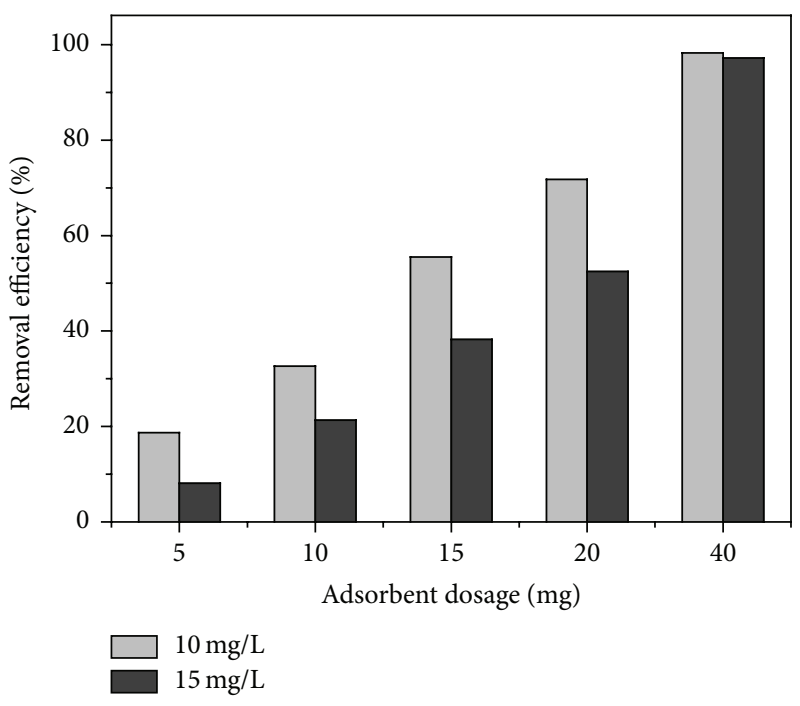

FIGURE 4: Effect of dosage of the CTAB-modified cornstalk biochar on ORII adsorption. The concentrations for ORII were 10 and $15 \mathrm{mg} / \mathrm{L}$, respectively.

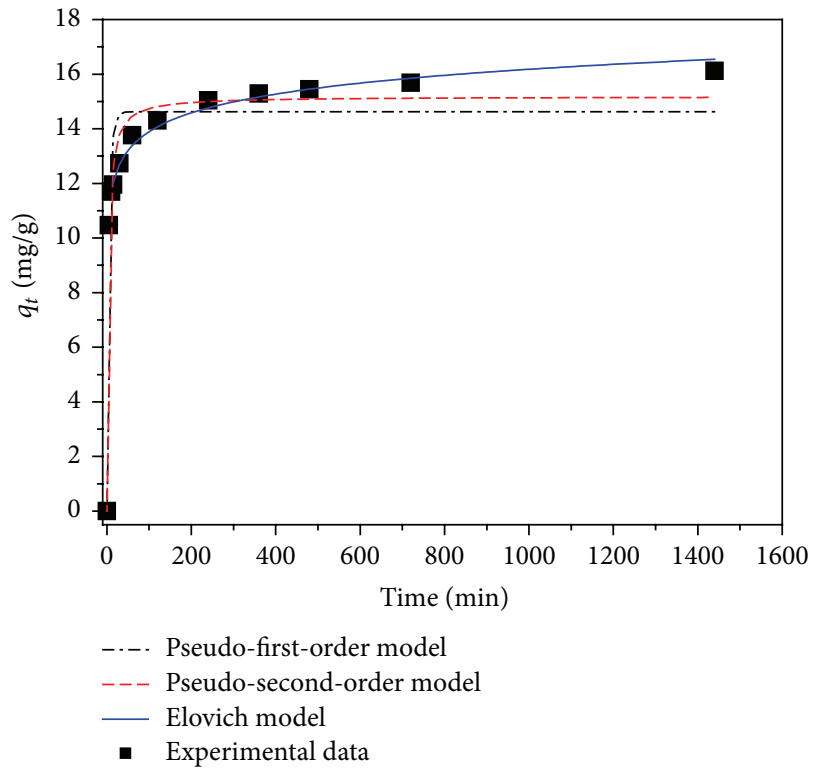

FIGURE 5: Nonlinear adsorption kinetics at pH 7.0 and fitted curves for ORII adsorption onto the CTAB-modified cornstalk biochar.

where $a$ and $b$ are the constants relating to the fraction of the surface covered and the chemisorption activation energy.

The adsorption process typically consists of an especially rapid initial uptake and a subsequent smooth increase to equilibrium within $24 \mathrm{~h}$. Using the nonlinear regressive method, the experimental kinetic data for ORII adsorption at $\mathrm{pH} 7.0$ were first simulated by pseudo-first-order, pseudosecond-order, and Elovich kinetic models (Figure 5). The parameters for the three models at $\mathrm{pH} 3.0,5.0,7.0,9.0$, and 11.0 are summarized in Table 1 for comparison. From Figure 5, it is evident that the kinetic curve simulated by the Elovich model is the best to describe the experimental points as 


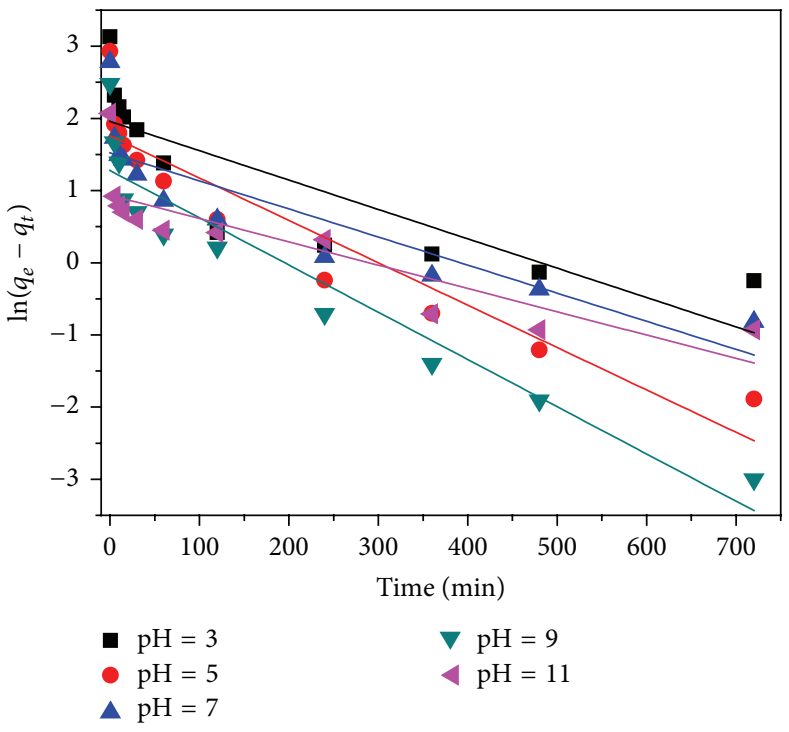

(a)

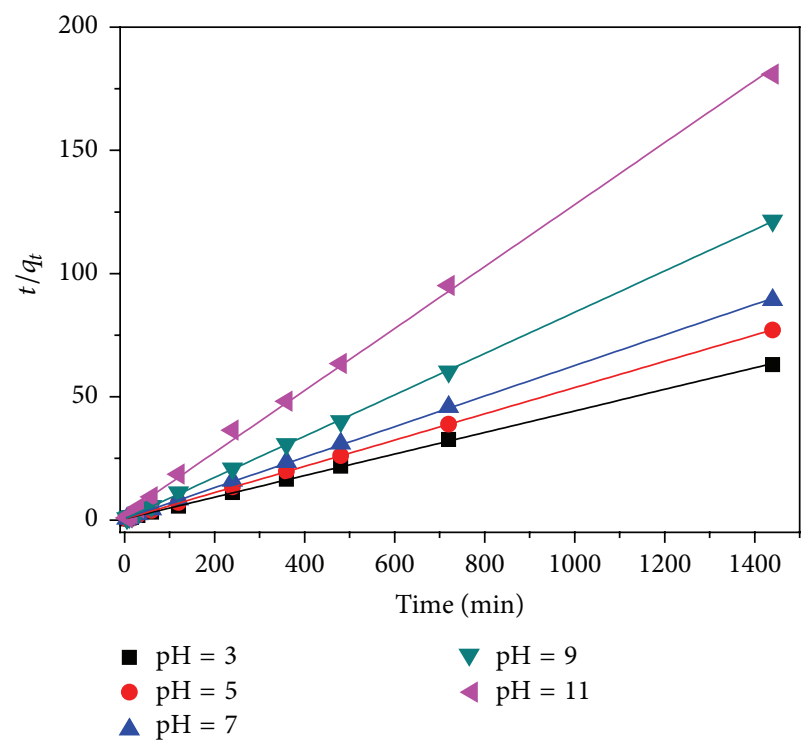

(b)

FIGURE 6: Linear adsorption kinetics for pseudo-first-order and pseudo-second-order simulation of ORII adsorption onto the CTABmodified cornstalk biochar.

TABle 1: Parameters for the nonlinear kinetic models including pseudo-first-order, pseudo-second-order, and Elovich models.

\begin{tabular}{lccccc}
\hline Kinetic model & $\mathrm{pH}=3$ & $\mathrm{pH}=5$ & $\mathrm{pH}=7$ & $\mathrm{pH}=9$ & $\mathrm{pH}=11$ \\
\hline $\begin{array}{l}\text { Pseudo-first-order } \\
\quad\end{array}$ & & & & & \\
$\quad k_{1}\left(\mathrm{~min}^{-1}\right)$ & 0.117 & 0.159 & 0.191 & 0.139 & 0.235 \\
$q_{e}(\mathrm{mg} / \mathrm{g})$ & 20.9 & 17.2 & 14.6 & 11.3 & 7.00 \\
$R^{2}$ & 0.903 & 0.901 & 0.922 & 0.956 & 0.901 \\
Pseudo-second-order & & & & & \\
$\quad k_{2}(\mathrm{~g} / \mathrm{mg} \cdot \mathrm{min})$ & 0.00866 & 0.0150 & 0.0221 & 0.0204 & 0.0569 \\
$q_{e}(\mathrm{mg} / \mathrm{g})$ & 21.9 & 17.9 & 15.2 & 11.7 & 7.3 \\
$R^{2}$ & 0.971 & 0.967 & 0.975 & 0.989 & 0.954 \\
Elovich & & & & & \\
$a$ & 10.34 & 9.997 & 9.325 & 6.360 & 4.679 \\
$k$ & 2.099 & 1.234 & 0.992 & 0.878 & 0.450 \\
$R^{2}$ & 0.981 & 0.992 & 0.996 & 0.968 & 0.998 \\
\hline
\end{tabular}

the experimental data are closest to the fitted Elovich curve. Further, judging from the values of regression coefficient $\left(R^{2}\right)$, it can be observed that the Elovich model fitted the kinetic data better than pseudo-first-order and pseudosecond-order kinetic models at all the $\mathrm{pH}$ conditions. Overall, the Elovich kinetic model could describe the kinetic data at all the solution $\mathrm{pH}$ examined by the nonlinear regressive method.

Concurrently, the experimental data for the adsorption kinetics were also fitted by the linear pseudo-first-order and pseudo-second-order kinetic models (Figure 6). Evidently, the experimental data were more in line with the fitted curves of the pseudo-second-order model than the pseudo-firstorder model. The values of regression coefficient $\left(R^{2}\right)$ at different $\mathrm{pH}$ conditions for the pseudo-first-order model are all less than 0.891 whereas those for the pseudo-second-order model were all bigger than 0.998. Moreover, the calculated $q_{e}$ values from pseudo-second-order model agreed better with the experimental data as well. The pseudo-second-order kinetic model is based on the assumption that the ratedetermining factor may be chemisorption involving electron sharing or transfer between adsorbent and adsorbate, while the Elovich model is better used to describe the adsorption kinetics of an ion exchange system [19]. Thus, it can be deduced that the ORII adsorption process on the CTABmodified cornstalk biochar might be a chemical adsorption process accompanied by an ion exchange $[12,16,20]$.

Additionally, as suggested by the aforementioned results, CTAB has overwhelmingly modified the surface charge property of the cornstalk biochar. However, the solution $\mathrm{pH}$ could influence the uptake of ORII on the CTAB-modified cornstalk biochar by altering the surface functional groups of sorbent and the dissociation of ORII molecules. The values of $\mathrm{p} K_{a 1}$ for the deprotonation of the naphthalene $\mathrm{OH}$ and $\mathrm{pK}_{a 2}$ for the deprotonation of $\mathrm{SO}_{3} \mathrm{H}$ group of ORII are 11.4 and $\sim 1$, respectively [21]. Accordingly, the ORII molecules are dominantly negatively charged from $\mathrm{pH} 3.0$ to 11.0. On the other hand, as the functional groups such as carboxylic and phenolic groups on the modified biochar are sensitive to the variation of solution $\mathrm{pH}$, the CTAB-modified cornstalk biochar is expected to be more positively charged under acidic conditions and more negatively charged under alkaline conditions. As a result, the uptake of ORII proved to be favorable under acidic conditions but unfavorable under alkaline conditions; these results are consistent with the results presented in Table 1 . In short, the electrostatic interaction is dominant for the whole adsorption process. 


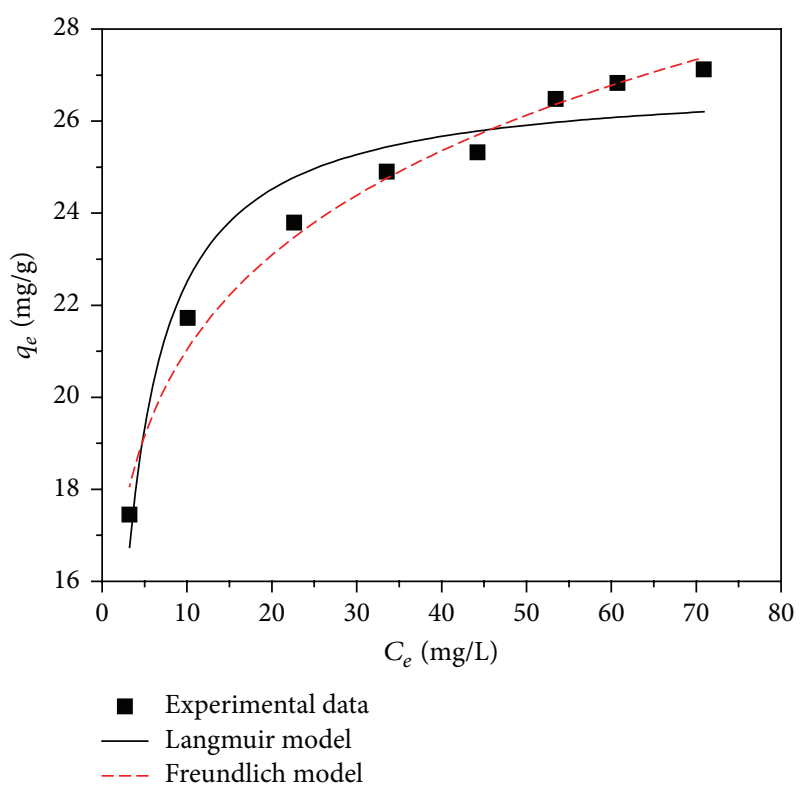

FIGURE 7: Adsorption isotherm at $298 \mathrm{~K}$ and fitted curves of ORII adsorption onto the CTAB-modified cornstalk biochar.

3.5. Adsorption Isotherm and Thermodynamics. In order to evaluate the adsorption capability of the modified biochar, the adsorption isotherm was investigated at 288, 298, and $308 \mathrm{~K}$, respectively. For simplicity, only the adsorption isotherm at $298 \mathrm{~K}$ is illustrated and simulated in Figure 7. Both the Langmuir and Freundlich models were used to fit the experimental data, and the parameters for both models are listed in Table 2.

The saturated monolayer Langmuir isotherm can be represented as [22]

$$
q_{e}=\frac{q_{m} k_{L} C_{e}}{1+k_{L} C_{e}},
$$

where $q_{e}$ is the amount of ORII adsorbed onto the modified biochar $(\mathrm{mg} / \mathrm{g}), C_{e}$ is the equilibrium concentration $(\mathrm{mg} / \mathrm{L})$, $q_{m}$ is the maximal adsorption capacity of the sorbent $(\mathrm{mg} / \mathrm{g})$, and $k_{L}$ is the equilibrium adsorption constant related to the affinity of binding sites $(\mathrm{L} / \mathrm{mg})$.

The Freundlich isotherm is an empirical equation describing adsorption on a heterogeneous surface. It is commonly described as [23]

$$
q_{e}=k_{F} C_{e}^{1 / n},
$$

where $k_{F}$ and $n$ are the Freundlich constants related to the adsorption capacity and adsorption intensity of the sorbent, respectively.

The adsorption isotherm can provide information about the surface properties and adsorption behavior of adsorbent. Judging from the experimental data and fitted isotherm curves in Figure 6, both the Langmuir and Freundlich models fitted the experimental data well, whereas the experimental data were closer to the curve simulated by the Freundlich model than that by Langmuir model. The regression coefficient values of Freundlich model at all the temperatures
TABLE 2: Parameters of the Langmuir and Freundlich isotherm models for the adsorption of ORII onto the CTAB-modified cornstalk biochar.

\begin{tabular}{lccc}
\hline Adsorption model & \multicolumn{3}{c}{ Temperature } \\
& $288 \mathrm{~K}$ & $298 \mathrm{~K}$ & $308 \mathrm{~K}$ \\
\hline Langmuir & & & \\
$\quad q_{\max }(\mathrm{mg} / \mathrm{g})$ & 25.4 & 26.9 & 29.1 \\
$k_{L}(\mathrm{~L} / \mathrm{mg})$ & 0.586 & 0.509 & 0.519 \\
$R^{2}$ & 0.911 & 0.932 & 0.923 \\
Freundlich & & & \\
$\quad k_{F}(\mathrm{mg} / \mathrm{g})$ & 15.38 & 15.43 & 16.44 \\
$n$ & 8.23 & 7.43 & 7.18 \\
$R^{2}$ & 0.985 & 0.982 & 0.976 \\
\hline
\end{tabular}

are higher than those of the Langmuir model, indicating the heterogeneous surface of the modified biochar. As presented in Table 2, by the Langmuir model, the calculated maximal adsorption capacities for phosphate achieved 25.4, 26.9 , and $29.1 \mathrm{mg} / \mathrm{g}$ at 288,298 , and $308 \mathrm{~K}$, respectively. It is evident that ORII adsorption increased with an increase in reaction temperatures, indicating the adsorption process was endothermic in nature.

The thermodynamic parameters associated with the adsorption mechanism including standard Gibbs free energy change $\left(\Delta G^{0}\right)$, standard enthalpy change $\left(\Delta H^{0}\right)$, and standard entropy change $\left(\Delta S^{0}\right)$ were calculated using the following equations:

$$
\begin{aligned}
\Delta G^{0} & =-R T \ln K_{0}, \\
\Delta G^{0} & =\Delta H^{0}-T \Delta S^{0}, \\
\ln K_{0} & =-\frac{\Delta H^{0}}{R T}+\frac{\Delta S^{0}}{R},
\end{aligned}
$$

where the thermodynamic equilibrium constant $K_{0}$ for the adsorption process was determined by plotting $\ln q_{e} / C_{e}$ versus $q_{e}$ and extrapolating to zero $q_{e}$ using a graphical method [24]. The intersection with the vertical axis gives the value of $\ln K_{0}$ at the three different temperatures. The enthalpy and entropy of the adsorption process are calculated to be $38.45 \mathrm{KJ} \mathrm{mol}^{-1}$ and $185.0 \mathrm{~J} \mathrm{~mol}^{-1} \mathrm{~K}^{-1}$, respectively. The positive value of the reaction enthalpy implies that the uptake of ORII increased with an increase in the reaction temperature; this result is consistent with the aforementioned results. The positive value of the enthalpy change also indicated that the adsorption process is endothermic. The negative values of $\Delta G^{0}-14.92,-16.50$, and $-18.62 \mathrm{KJ} \mathrm{mol}^{-1}$ at 288,298 , and $308 \mathrm{~K}$, respectively, suggested the spontaneous nature of ORII adsorption.

\section{Conclusion}

The composite adsorbent, CTAB-modified cornstalk biochar, was successfully prepared and used for the removal of negatively charged pollutants such as Orange II. Compared to the virgin cornstalk biochar, the modified biochar demonstrated 
its excellent adsorption capability for Orange II removal. The uptake of Orange II increased with a decrease of solution $\mathrm{pH}$. The electrostatic interaction proved to be dominant for the uptake of the dye. Kinetic experiments indicated that the ORII adsorption process on the CTAB-modified cornstalk biochar might be chemical adsorption accompanied by ion exchange. Thermodynamic analysis indicated that the adsorption process is spontaneous and endothermic. A large amount of CTAB proved to be still combined with the stable substrate biochar after the adsorption process. These results suggest that the CTAB-modified cornstalk biochar is a promising candidate for the removal of negatively charged pollutants.

\section{Competing Interests}

The authors declare that they have no competing interests.

\section{Acknowledgments}

The authors appreciate the finical support from the National Science Foundation of China (Grant no. 51378205) and the Foundation for University Key Youth Teacher of Henan Province of China (2013GGJS-088).

\section{References}

[1] T. Robinson, G. McMullan, R. Marchant, and P. Nigam, "Remediation of dyes in textile effluent: a critical review on current treatment technologies with a proposed alternative," Bioresource Technology, vol. 77, no. 3, pp. 247-255, 2001.

[2] P. Westerhoff, Y. Yoon, S. Snyder, and E. Wert, "Fate of endocrine-disruptor, pharmaceutical, and personal care product chemicals during simulated drinking water treatment processes," Environmental Science and Technology, vol. 39, no. 17, pp. 6649-6663, 2005.

[3] J. H. Qu, "Research progress of novel adsorption processes in water purification: a review," Journal of Environmental Sciences, vol. 20, no. 1, pp. 1-13, 2008.

[4] S. Sadaf, H. N. Bhatti, S. Nausheen, and S. Noreen, "Potential use of low-cost lignocellulosic waste for the removal of direct violet 51 from aqueous solution: equilibrium and breakthrough studies," Archives of Environmental Contamination and Toxicology, vol. 66, no. 4, pp. 557-571, 2014.

[5] Y. Yu, C. Wang, X. Guo, and J. P. Chen, "Modification of carbon derived from Sargassum sp. by lanthanum for enhanced adsorption of fluoride," Journal of Colloid and Interface Science, vol. 441, pp. 113-120, 2015.

[6] S. Babel and T. A. Kurniawan, "Low-cost adsorbents for heavy metals uptake from contaminated water: a review," Journal of Hazardous Materials, vol. 97, no. 1-3, pp. 219-243, 2003.

[7] S. Sadaf, H. N. Bhatti, S. Nausheen, and M. Amin, "Application of a novel lignocellulosic biomaterial for the removal of Direct Yellow 50 dye from aqueous solution: Batch and column study," Journal of the Taiwan Institute of Chemical Engineers, vol. 47, pp. 160-170, 2015.

[8] H. N. Bhatti and S. Nausheen, "Equilibrium and kinetic modeling for the removal of Turquoise Blue PG dye from aqueous solution by a low-cost agro waste," Desalination and Water Treatment, vol. 55, no. 7, pp. 1934-1944, 2015.
[9] T. Maruyama, Y. Terashima, S. Takeda, F. Okazaki, and M. Goto, "Selective adsorption and recovery of precious metal ions using protein-rich biomass as efficient adsorbents," Process Biochemistry, vol. 49, no. 5, pp. 850-857, 2014.

[10] J. W. Lee, B. Hawkins, D. M. Day, and D. C. Reicosky, "Sustainability: the capacity of smokeless biomass pyrolysis for energy production, global carbon capture and sequestration," Energy and Environmental Science, vol. 3, no. 11, pp. 1695-1705, 2010.

[11] J. Lehmann, "A handful of carbon," Nature, vol. 447, no. 7141, pp. 143-144, 2007.

[12] R. Zhang, J. Zhang, X. Zhang, C. Dou, and R. Han, "Adsorption of Congo red from aqueous solutions using cationic surfactant modified wheat straw in batch mode: kinetic and equilibrium study," Journal of the Taiwan Institute of Chemical Engineers, vol. 45, no. 5, pp. 2578-2583, 2014.

[13] H. Kavas, Z. Durmus, M. Şenel, S. Kazan, A. Baykal, and M. S. Toprak, "CTAB- $\mathrm{Mn}_{3} \mathrm{O}_{4}$ nanocomposites: synthesis, NMR and low temperature EPR studies," Polyhedron, vol. 29, no. 5, pp. 1375-1380, 2010.

[14] M. Ahmad, A. U. Rajapaksha, J. E. Lim et al., "Biochar as a sorbent for contaminant management in soil and water: a review," Chemosphere, vol. 99, pp. 19-23, 2014.

[15] S. Lagergren, "Zur theorie der sogenannten adsorption gelöster stoffe. Kungliga Svenska Vetenskapsakademiens," Handlinga, vol. 24, no. 4, pp. 1-39, 1898.

[16] Y. S. Ho and G. McKay, "Pseudo-second order model for sorption processes," Process Biochemistry, vol. 34, no. 5, pp. 451$465,1999$.

[17] M. Kithome, J. W. Paul, L. M. Lavkulich, and A. A. Bomke, "Kinetics of ammonium adsorption and desorption by the natural zeolite clinoptilolite," Soil Science Society of America Journal, vol. 62, no. 3, pp. 622-629, 1998.

[18] C. W. Cheung, J. F. Porter, and G. McKay, "Sorption kinetics for the removal of copper and zinc from effluents using bone char," Separation and Purification Technology, vol. 19, no. 1-2, pp. 5564, 2000.

[19] Y. S. Ho, J. C. Y. Ng, and G. McKay, "Kinetics of pollutant sorption by biosorbents: review," Separation and Purification Methods, vol. 29, no. 2, pp. 189-232, 2000.

[20] N. Gupta, A. K. Kushwaha, and M. C. Chattopadhyaya, "Adsorption studies of cationic dyes onto Ashoka (Saraca asoca) leaf powder," Journal of the Taiwan Institute of Chemical Engineers, vol. 43, no. 4, pp. 604-613, 2012.

[21] J. Bandara, J. A. Mielczarski, and J. Kiwi, "1. Molecular mechanism of surface recognition. Azo dyes degradation on $\mathrm{Fe}, \mathrm{Ti}$, and Al oxides through metal sulfonate complexes," Langmuir, vol. 15 , no. 22, pp. 7670-7679, 1999.

[22] H. M. F. Freundlich, "Uber die adsorption in lasungen," Journal of Physical Chemistry, vol. 57, pp. 385-470, 1906.

[23] S. Yang, D. Ding, Y. Zhao et al., "Investigation of phosphate adsorption from aqueous solution using Kanuma mud: behaviors and mechanisms," Journal of Environmental Chemical Engineering, vol. 1, no. 3, pp. 355-362, 2013.

[24] X. Yuan, W. Xing, S.-P. Zhuo et al., "Preparation and application of mesoporous Fe/carbon composites as a drug carrier," Microporous and Mesoporous Materials, vol. 117, no. 3, pp. 678-684, 2009. 

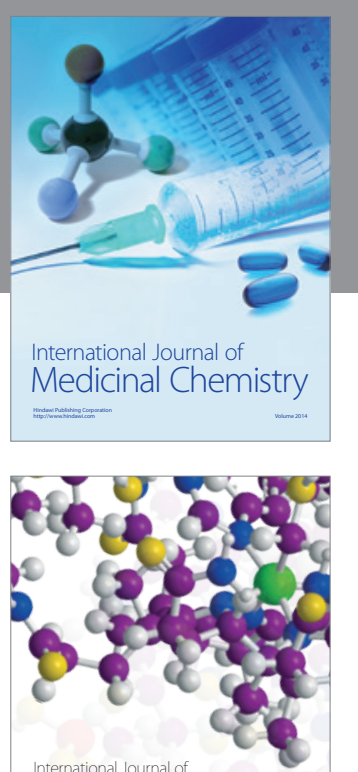

Carbohydrate Chemistry

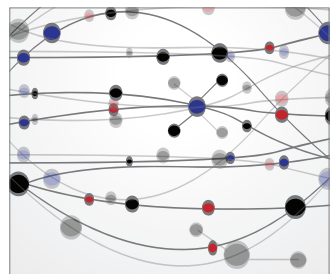

The Scientific World Journal
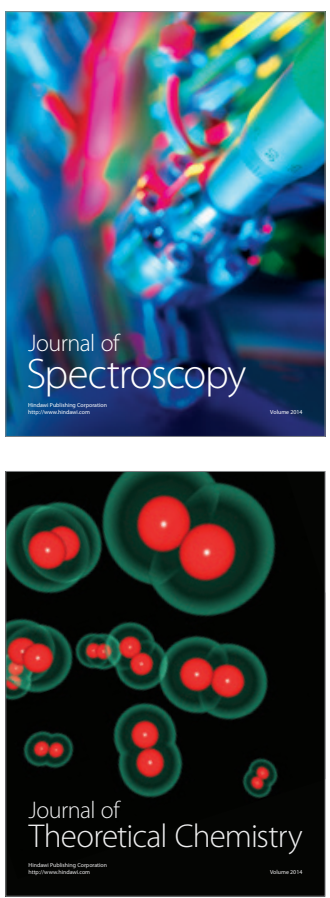
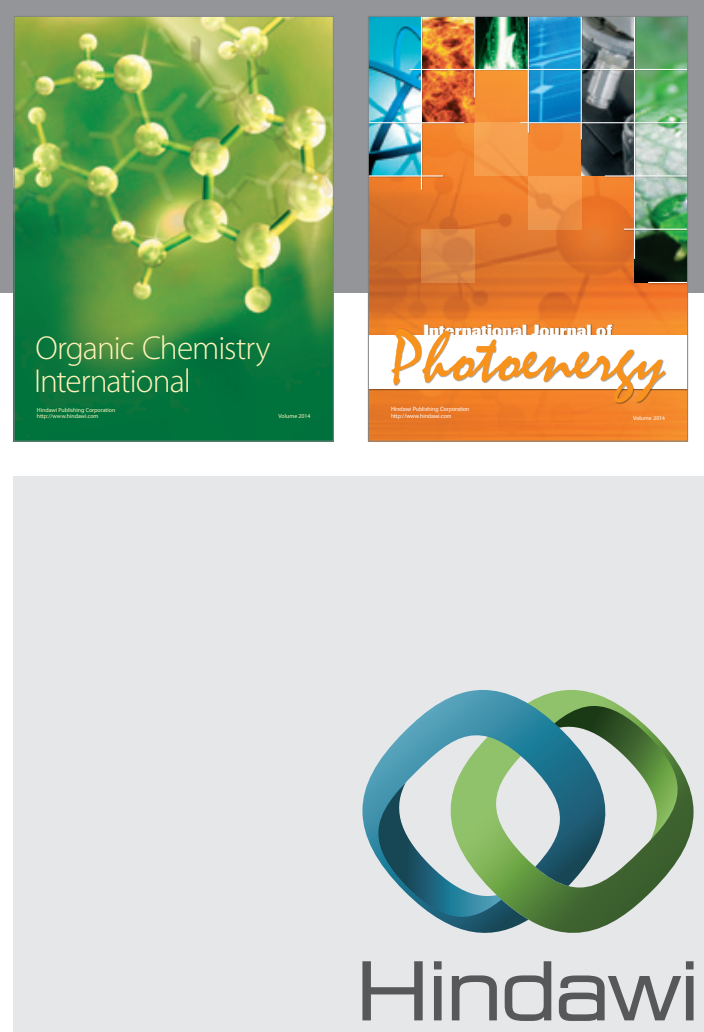

Submit your manuscripts at

http://www.hindawi.com

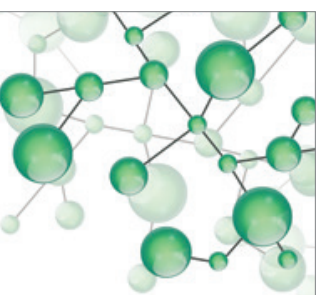

International Journal of

Inorganic Chemistry

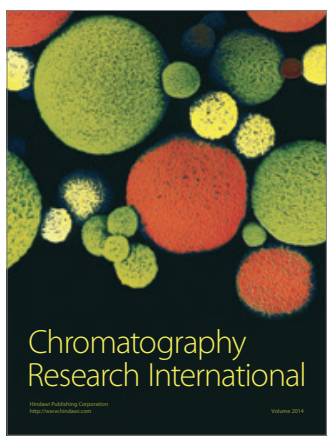

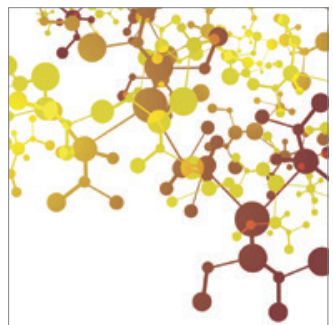

Applied Chemistry
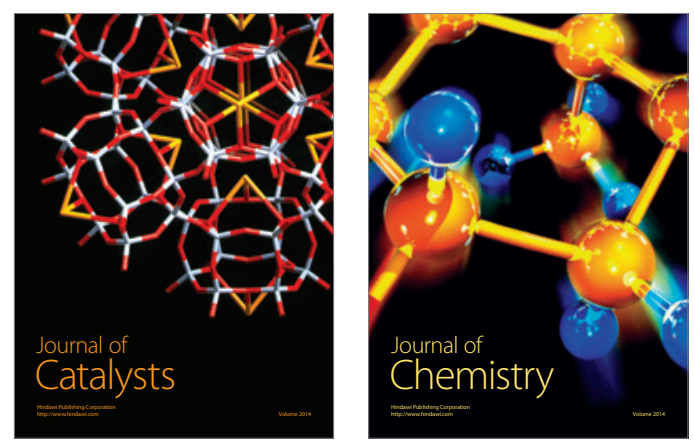
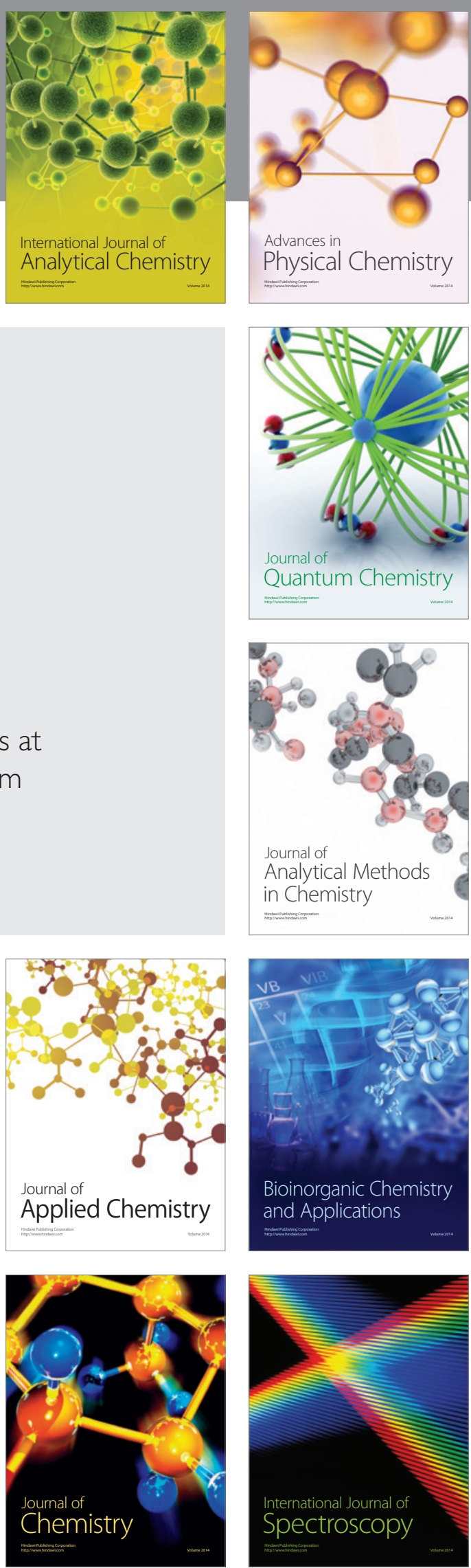